\title{
Combinational approach using solid dispersion and semi-solid matrix technology to enhance in vitro dissolution of telmisartan
}

\author{
Syed Faisal Ali ${ }^{1}$, Monika Joshi ${ }^{1}$, Nida Akhtar ${ }^{1}$, Vijay Sharma ${ }^{1}$, Kamla Pathak ${ }^{2 *}$ \\ ${ }^{1}$ Department of Pharmaceutics, Rajiv academy for pharmacy, Mathura, India \\ ${ }^{2}$ Department of Pharmaceutics, Pharmacy College Saifai, Saifai, Etawah, Uttar Pradesh, India \\ Received: Feb 22, 2016, Revised: Mar 3, 2016, Accepted: Mar 26, 2016
}

\begin{abstract}
The present investigation was focused to formulate semi-solid capsules (SSCs) of hydrophobic drug telmisartan (TLMS) by encapsulating semi-solid matrix of its solid dispersion (SD) in HPMC capsules. The combinational approach was used to reduce the lag time in drug release and improvise its dissolution. SDs of TLMS was prepared using hot fusion method by varying the combinations of Pluronic-F68, Gelucire 50/13 and Plasdone S630. A total of nine batches (SD1-SD9) were characterized for micromeritic properties, in vitro dissolution behavior and surface characterization. SD4 with $52.43 \%$ cumulative drug release (CDR) in phosphate buffer, $\mathrm{pH} 7.4$, in $120 \mathrm{~min}, \mathrm{t}_{50 \%} 44.2 \mathrm{~min}$ and $\mathrm{DE}_{30 \min } 96.76 \%$ was selected for the development of semi-solid capsules. Differential scanning calorimetry of SD4 revealed molecular dispersion of TLMS in Pluronic-F68. SD4 was formulated into SSCs using Gelucire 44/14 and PEG 400 as semi-solid components and PEG 6000 as a suspending agent to achieve reduction in lag time for effective drug dissolution. SSC6 showed maximum in vitro drug dissolution $97.49 \%$ in phosphate buffer, $\mathrm{pH} 7.4$ with in 20 min that was almost a three folds reduction in the time required to achieve similar dissolution by SD. Thus, SSCs present an excellent approach to enhance in vitro dissolution as well as to reduce the lag time of dissolution for poorly water soluble drugs especially to those therapeutic classes that are intended for faster onset of action. Developed approach based on HPMC capsules provided a better alternative to target delivery of telmisartan to the vegetarian population.
\end{abstract}

Keywords: in vitro dissolution, lag time, HPMC capsules, solid dispersion, solubility

Pharm Biomed Res 2016; 2(1): 23-35

DOI: 10.18869/acadpub.pbr.2.1.23

\section{Introduction}

Telmisartan (TLMS) is an angiotensin II receptor antagonist employed for its anti-arrhythmic, anti-anginal and antihypertensive activity (1). One of the major problems related with it is the low solubility in biological fluids, which results into poor bioavailability after oral administration $(\sim 42 \%)$ and late onset of action. It also shows high first pass metabolism which further reduces the oral bioavailability (2).
It is a very slightly water soluble drug having aqueous solubility of 0.078 $\mathrm{mg} / \mathrm{ml}$. Limited aqueous solubility leads to dissolution rate limited absorption and subsequently low bioavailability (3). Any lag time due to its poor dissolution will result in late onset of action and may clinically present an emergency situation. Thus, increasing its aqueous solubility and dissolution behavior is of therapeutic importance. 
Telmisartan loaded with mesoporous silica nanoparticles (MSNs) were formulated by Zhang et al to improve the dissolution rate and enhance the drug loading capacity. The drug release rate from MSNs with a pore diameter of $12.9 \mathrm{~nm}$ was found to be effectively increased and the drug release rate from the functionalized MSNs was effectively controlled compared with that from the unmodified MSNs. It was depicted by the authors MSNs help in the design of oral drug delivery systems for the dissolution enhancement and/or sustained release of poorly watersoluble drugs (4). Further, Patel et al. had increased the solubility Telmisartan by using Surface solid dispersion (SSD) made of polymers like Poloxamer 407, PEG 6000 and aerosil 200 as adsorbent. Telmisartan adsorbed over carrier and adsorbent resulting in a higher surface area of drug exposed to the dissolution media and improved wettability of the drug particles. The significant reduction in particle size during the formation of SSDs and the inherently higher rate of dissolution of the soluble component of SDs may also contribute to the increased solubility of Telmisartan (2). However, the total pore volume and the pore diameter of MSNs were the two main factors limiting the maximum drug load capacity, thus affecting the drug release.

Techniques like cyclodextrin complexation, solid dispersion, nanoparticles have been used in order to enhance the solubility and hence bioavailability (3,5-6). Out of these techniques, solid dispersion is considered to be simple, easy to optimize, reproducible and a promising approach for formulators (7). Other techniques like filling of semi solid and liquid matrices into hard gelatin capsules are commonly used for enhancing solubility of poorly soluble drugs (8).

Kumar et al had reported liquid fill technique in hard gelatin capsules using Gelucire and their blends, an efficacious approach for designing immediate or sustained drug release profiles for poorly soluble drugs like aceclofenac. The in vivo study of the optimized immediate release and sustained release formulations revealed significant enhancement in anti inflammatory activity (9). Further, Alladi and Shastri had also demonstrated the enhanced dissolution and therapeutic efficacy of BCS class-II drugs (meloxicam and tenoxicam) by lipid semisolid matrix (10). These methodologies have several advantages viz weight and content uniformity, development and establishment of dust free manufacturing process (8). Additionally, the type and amount of carrier can control the semisolid preparation (at room temperature) and presents easy dust free transportation. On the other hand, temperature higher than room temperature provides flowability to the matrix thus making capsule filling easy (11). An in-house research report based on semi-solid capsules (SSCs) depicted enhanced in vitro dissolution of amlodipine besilate and almost three times reduction in the time required by solid dispersion (SD) to achieve similar percent cumulative drug release (CDR) (12). Keeping in view the advantageous potential of the technology and the limitations associated with the drug, the project was undertaken.

Thus, the project was aimed to develop SSC of TLMS that would enhance the solubility of the active ingredient, improvise in vitro dissolution, reduce 
the lag time, and hence enhance the therapeutic efficacy. Further, the usage of hydroxyl propyl methyl cellulose (HPMC) empty capsule shells provides additional advantage to the developed system, as HPMC based capsules are reported to be purely $(100 \%)$ vegetarian (13). HPMC capsules prepared from HPMC (hypermellose) developed by synthetic modification of natural polymer cellulose and is reported to be as safe for human consumption may provide attractive alternative to gelatin capsules because of its vegetable source (14). These capsules exhibited physical characteristics either superior or similar as compared to the gelatin capsules (15). Further, HPMC based capsules have depicted rapid and comparable in vivo disintegration times with reference to gelatin and overcome some of the disadvantages of gelatin capsules present. As gelatin becomes brittle when stored at low humidity and shell hydration may occur with hygroscopic fillings. HPMC capsule shells can demonstrate lack of brittleness even at moisture levels below $2 \%$, no crosslinking and improved chemical stability (16-17). Considering these benefits, the project was undertaken to improvise the drug dissolution using HPMC based capsules which provided a better alternative to target delivery of telmisartan to the vegetarian population.

\section{Materials and methods \\ Materials}

TLMS was obtained as a gift sample from FDC pharmaceutical Ltd, Varena, Goa (India). Pluronic-F68 and Plasdone-S630 were procured from BASF Corporation, (U.S.A) and International Specialty Product
Technologies limited, U.S.A., respectively. Gelucire-50/13 and Gelucire 44/14 were procured from Colorcon Asia Pvt. Ltd. Mumbai, India. PEG 400 and PEG 6000 were procured from Central Drug House, New Delhi, India. HPMC capsule shell was gifted by ACG Associates Capsules Ltd, Mumbai, India.

\section{Solid dispersions preparation}

A total of nine formulations were developed as per the design (Table 1). Polymers (Plasdone-f68, Plasdone-S630 and Gelucire 50/30) were individually melted in separate china dish above their melting points, and the weighed amount of drug was gradually incorporated into the molten polymer. The mixtures were cooled with constant stirring to homogenously disperse the drug. Cooled mass was then crushed in a glass mortar, passed through sieve No.22 and stored in a desiccator till further use.

\section{Powder properties of SDs}

Prepared granules were evaluated for various micromeritic parameters like angle of repose, bulk density, tapped density, Carr's index and Hausner's ratio. Dynamic angle of repose was determined by placing 1 $\mathrm{g}$ of powder in a lab fabricated rotating cylinder apparatus and allowed to rotate at $25 \mathrm{rpm}$ for 5 min. The angle made by the bulk of the drug powder against the horizontal tangent was recorded and dynamic angle of repose each sample was calculated (18). The bulk density was obtained by dividing the weight of sample by the final volume in $\mathrm{cm}^{3}$ of the sample contained in the cylinder (19). Hausner's ratio was 


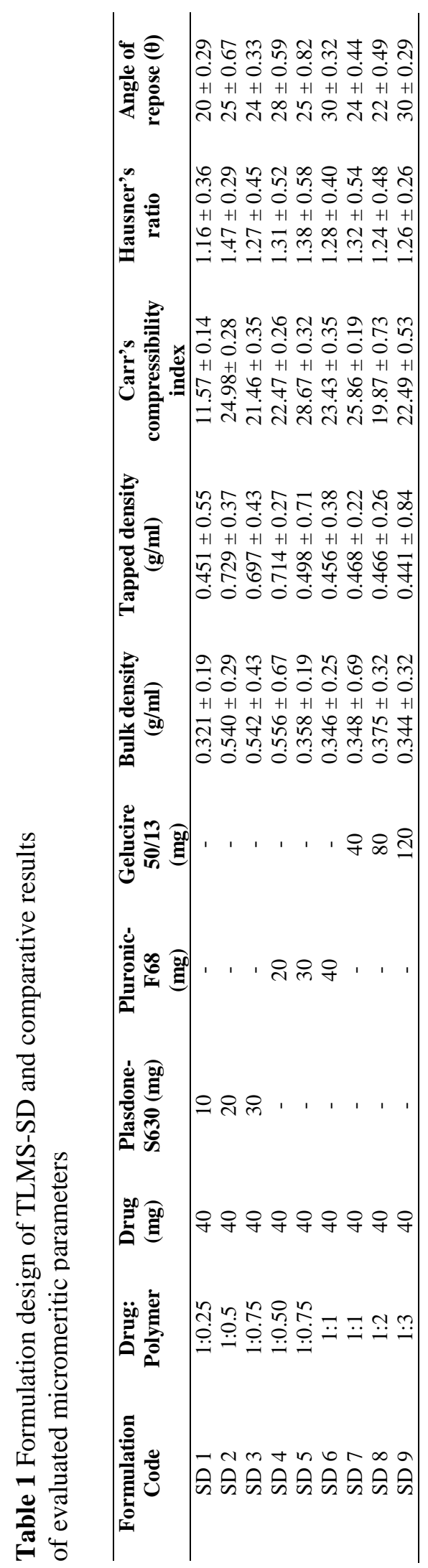

determined by dividing the tapped density by bulk density. The percent compressibility index was calculated by using the equation:

$\%$ Compressibility Index $=$ (Tapped density-Poured density)/Tapped density $\times 100$

\section{In vitro dissolution}

In vitro dissolution of pure drug, its physical mixture and SDs (drug equivalent to $40 \mathrm{mg}$ ) was carried out using USP XXIII apparatus II containing $900 \mathrm{ml}$ of phosphate buffer, $\mathrm{pH} 7.4$ at an agitation rate of $100 \mathrm{rpm}$. The temperature of the medium was maintained at $37 \pm 0.5{ }^{\circ} \mathrm{C}$. Five milliliters of sample was withdrawn at regular intervals $(0,5,10,15,20,30,40$, 50 , and $60 \mathrm{~min}$ ) and replaced with equal volume of fresh dissolution medium (sink conditions maintained), filtered and analyzed spectrophotometrically at $296 \mathrm{~nm}$.

\section{Analysis of in vitro drug dissolution}

Model independent parameters were calculated by PCP Disso 2.0, Pune, India (20). Percent dissolution efficiency $(\% \mathrm{DE})$ was computed to compare the relative performance of polymers in solid dispersions. The magnitude of \%DE for each solid dispersion was computed as the percent ratio of area under the dissolution curve up to time t, to that of the area of the rectangle described by $100 \%$ dissolution at the same time (21).

X-Ray Powder Diffraction (XRPD)

XRPD was performed using a D/Max2400 X-ray Fluorescence Spectrometer (Rigaku, Japan) with $\mathrm{Cu} \mathrm{Ka}$ lines as a source of radiation. Standards run using a voltage of $56 \mathrm{KV}$, a current of 182 $\mathrm{mA}$ and a scanning rate of $0.2 / \mathrm{min}$ over 
a $2 \theta$ range of $0-70$ were carried out. Analysis was carried out for pure TLMS, Pluronic-F68, TLMS-SD and physical mixture of TLMS.

\section{Scanning Electron Microscopy (SEM)} The surface morphology of pure drug, carrier, physical mixture and solid dispersion was examined using FEI Quanta 200 scanning electron microscope (FEI Co., Netherlands, Europe). The samples were fixed on a brass stub using a double sided tape, gold coated in a vacuum by sputter coater and examined at a magnification of $100 x$.

\section{Differential Scanning Calorimetry (DSC)}

Differential Scanning Calorimetric thermograms of TLMS, Pluronic-F68, physical mixture and optimized formulation were obtained with DSC-60 controlled by TA-60 WS software (Shimadzu, Kyoto, Japan). Samples were weighed and transferred to the equipment for analysis in sealed hermetically sealed aluminum pans. Instrument was equipped with an intracooler in order to assess the change in chemical properties of samples. An indium standard was used to calibrate the differential scanning calorimeter temperature. Heating was done at a rate $10^{\circ} \mathrm{C} / \mathrm{min}$, over temperature range of 0 $300{ }^{\circ} \mathrm{C}$ and inert temperature was maintained by nitrogen purging at a rate of $10 \mathrm{ml} / \mathrm{min}(22)$.

Diffuse Reflectance Spectroscopy (DRS) DRS spectra were obtained after appropriate background subtraction using FTIR spectrophotometer (FTIR8400SCE, Shimadzu Corporation, Tokyo, Japan) equipped with a diffuse reflectance accessory (DRS-8000,
Shimadzu Corporation) and a datastation. Previously dried powder samples of pure drug and SD4 were mixed with dry potassium bromide and scanned within the range of 4000 to 400 $\mathrm{cm}^{-1}$.

\section{Semi solid capsules preparation}

Semisolid matrix of optimized solid dispersion formulation (SD4) was prepared by melting method. Semi solid polymers (Gelucire-44/14, PEG 4000) and suspending agent (PEG 4000) were melted and a homogenous matrix was prepared. The mixture was heated on a water bath at $80{ }^{\circ} \mathrm{C}$ with continuous stirring to obtain a homogenous melt. To this mixture, optimized SD equivalent to $10 \mathrm{mg}$ of drug was gradually incorporated. Prepared semi solid matrices were individually poured into preheated plastic injector and calculated amount (equivalent to $45 \mathrm{mg}$ ) of TLMS was transferred in HPMC capsule (size 0) shell. To ensure the efficient flow of dispersion into capsules, the filling step must be carried out at a temperature above the melting point of PEG 6000. A total of seven batches of SSCs were prepared (Table 2).

\section{Dispersal homogeneity}

To ensure the homogeneity of a semisolid mix (in turn, homogeneity of drug within the system) before filling into the capsules, dispersal homogeneity test was performed. A weighed quantity of the semisolid mix prepared by incorporating solid dispersion was transferred into the test tube. Mixture was then mixed with $10 \mathrm{ml}$ of distilled water and centrifuged (4000 rpm) for 10 min. Homogeneity of the material was examined visually for any sediment at the bottom of the tube and floating 
Table 2 Formulation design for semi solid capsule of telmisartan SD

\begin{tabular}{ccccc}
\hline Formulation code & $\begin{array}{c}\text { SD4 } \\
(\mathbf{m g})\end{array}$ & $\begin{array}{c}\text { PEG 400 } \\
(\mathbf{m g})\end{array}$ & $\begin{array}{c}\text { Gelucire 44/14 } \\
(\mathbf{m g})\end{array}$ & $\begin{array}{c}\text { PEG 6000 } \\
(\mathbf{m g})\end{array}$ \\
\hline SSC 1 & 45 & 50 & 50 & 20 \\
SSC 2 & 45 & 50 & 100 & 20 \\
SSC 3 & 45 & 100 & 50 & 10 \\
SSC 4 & 45 & 50 & 150 & 20 \\
SSC 5 & 45 & 150 & 50 & 10 \\
SSC 6 & 45 & 50 & 200 & 20 \\
SSC 7 & 45 & 200 & 50 & 10 \\
\hline
\end{tabular}

residue on the top layer of the sample. Additionally, $1 \mathrm{ml}$ volume was sampled was withdrawn from the top and bottom of the tubes. Drug content in each portion (top as well as bottom layer) was determined spectrophotometrically at $296 \mathrm{~nm}$.

\section{In vitro dissolution}

In vitro drug release was carried out using USP XXIII apparatus II containing $900 \mathrm{ml}$ phosphate buffer, $\mathrm{pH}$ 7.4. Temperature of the medium was maintained at $37 \pm 0.5{ }^{\circ} \mathrm{C}$. A $5 \mathrm{ml}$ sample was withdrawn at the sampling time points $(0,5,10,15$ and $20 \mathrm{~min})$ and replaced with the fresh media to maintain the sink conditions. The study was done in triplicate and samples were analyzed spectrophotometrically at 296 $\mathrm{nm}$. The release profiles were analyzed by model independent parameters calculated by PCP Disso 2.0 v (Pune, India) (20).

\section{Results}

Solid dispersion of telmisartan was formulated and subjected to powder characterization studies. Bulk density (poured) of solid dispersion granules prepared by different polymers was found to be in the range of $0.321 \pm 0.19$ $\mathrm{g} / \mathrm{ml}$ to $0.556 \pm 0.67 \mathrm{~g} / \mathrm{ml}$. While tapped density range from $0.441 \pm 0.84 \mathrm{~g} / \mathrm{ml}$ to $0.729 \pm 0.37 \mathrm{~g} / \mathrm{ml}$. As, angle of repose determined for all the powders displayed the value in the range of 20$25^{\circ} \mathrm{C}$. Flow of all the powders was also determined by calculating Carr's compressibility index. From the values, it was concluded that all the mixtures showed \% compressibility index between 11-21. Hausner's ratio is another parameter which depicted the flow properties of powders. It was observed that all the powders exhibited the value close to 1.2 or less (Table 1).

\section{In vitro dissolution}

In vitro dissolution of pure TLMS in phosphate buffer, $\mathrm{pH} 7.4$ documented 11.74 percent drug dissolution. Dose/solubility ratio for pure drug telmisartan, its solid dispersion (SD4) and semi solid capsule (SSC6) was calculated and found to be 423.728 , $216.452, \quad 117.370$, respectively in phosphate buffer, $\mathrm{pH}$ 7.4. According to current FDA guidance, the cut off value of $\mathrm{D} / \mathrm{S}$ ratio above 250 indicates that dissolution and absorption related problems may occur (23). For both, solid dispersion and semi solid capsule reduction of $\mathrm{D} / \mathrm{S}$ ratio was observed to be less in comparison to the pure drug telmisartan. Formulation of semi solid matrix from solid dispersion further reduced the $\mathrm{D} / \mathrm{S}$ ratio and thus the superiority of semi solid capsule over solid dispersion was revealed (24). From in vitro drug dissolution studies, cumulative amount drug release (in 120 


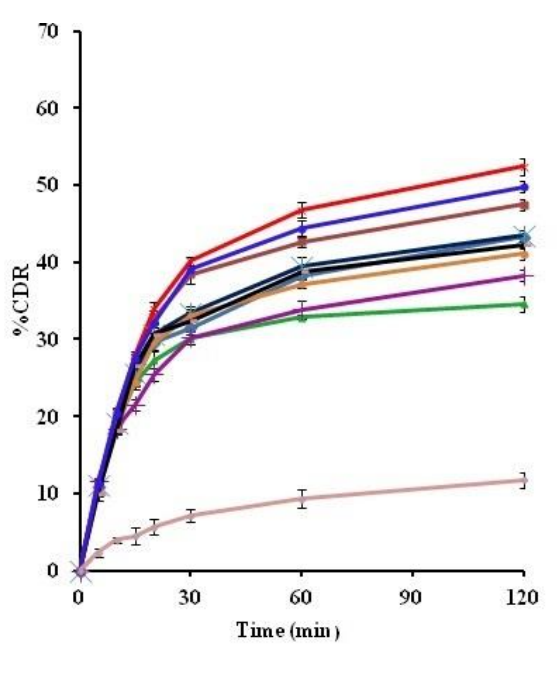

(A)

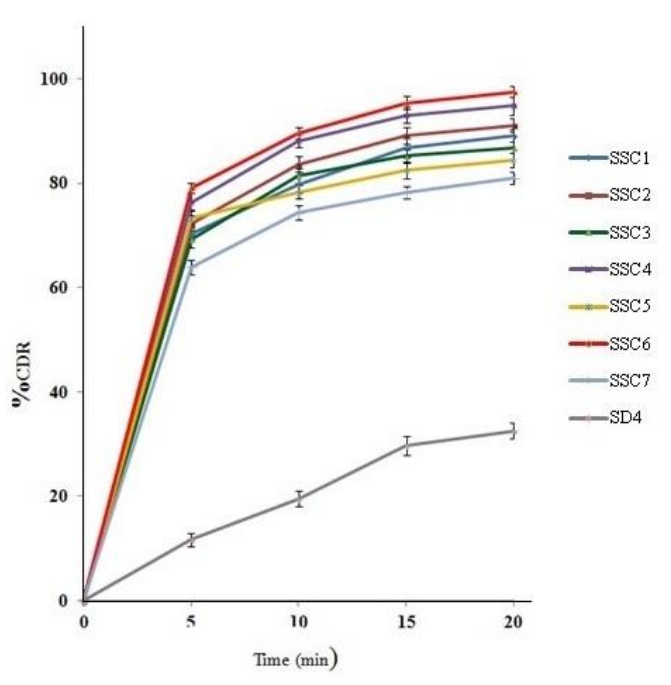

(B)

Figure 1 (A) Comparative in vitro dissolution profile of SD in phosphate buffer, $\mathrm{pH}$ 7.4. (B) In vitro drug dissolution profile of SSC in phosphate buffer, $\mathrm{pH} 7.4$

min) was found to be significantly higher in case of solid dispersion of telmisartan $(\mathrm{P}<0.05)$ as compared to the pure drug (Fig. 1A). Drug release increased with increase in ratio of drug: Plasdone-S630 from 1:0.25 to 1:0.50. However, when their ratio was $1: 0.75$, decrease in drug dissolution was observed. SD displayed an increase in \% CDR on increase in ratio of drug: PluronicF68 $(1: 0.50<1: 0.75<1: 1)$. Solid dispersion exhibited a higher dissolution of $52.43 \%$ of TLMS in phosphate buffer, $\mathrm{pH} 7.4$ within 120 min. Solid dispersion formulated using Gelucire-50/13 showed dissolution enhancement with increment in the drug: polymer ratio $(1: 1<1: 2<1: 3)$. A higher dissolution of about $49.81 \%$ of TLMS in phosphate buffer, $\mathrm{pH} 7.4$ was observed within 120 min which may be attributed to decrease in crystallinity and increase in wettability of the drug (25). itro drug dissolution data

Model independent parameters (\%DE and $\% \mathrm{~T}$ ) were obtained from cumulative $\%$ drug dissolution. Dissolution efficiency at $30 \mathrm{~min}$ and 60 min was found to be highest for SD4 i.e. 96.76 and 95.48, respectively in phosphate buffer, $\mathrm{pH}$ 7.4. The time required for $25 \%$ and $50 \%$ release was found to be least in SD4 i.e. $37.1 \mathrm{~min}$ and $44.1 \mathrm{~min}$ in phosphate buffer, $\mathrm{pH}$ 7.4. From all the parameters being evaluated, SD4 was considered as an optimized batch.

\section{$X$-Ray powder diffraction}

Among the TLMS-SD formulations, SD4 and its physical mixture were studied by XRD. Pure TLMS and Pluronic-F68 were also tested. XRPD was used to confirm the loss of drug crystallinity. The results are shown in Figure 2 A. Pure TLMS has several dominant peaks at 2-theta angles within 30 degrees. The crystallinity index for TLMS was found to be $83.56 \%$. In the physical mixture, although similar 


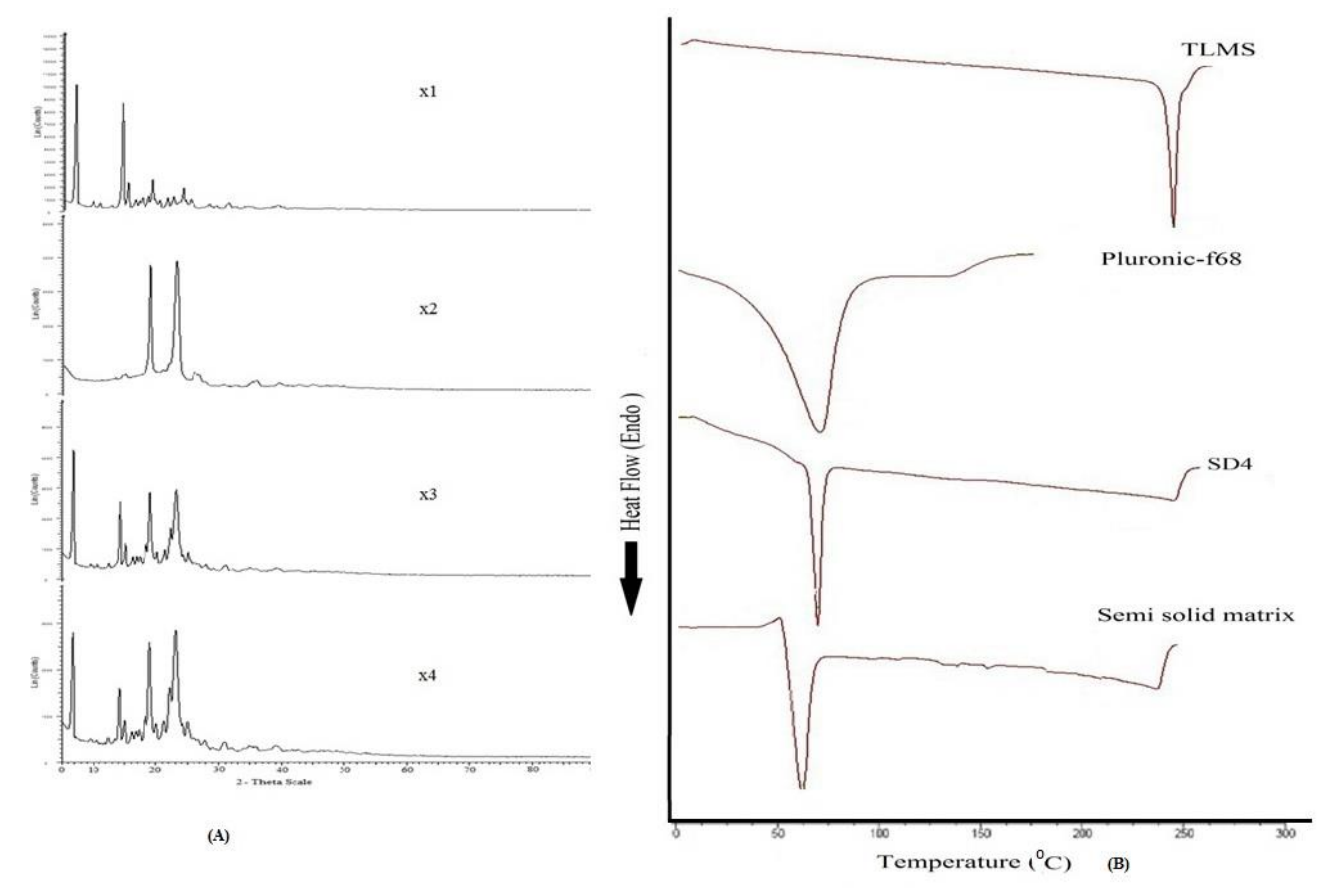

Figure 2 (A) XRPD patterns: X1) Pure TLMS, X2) Pluronic-F68, X3) physical mixture and X4) SD4. (B) Differential Scanning calorimetric thermograms of TLMS, Pluronic-F68, SD4 and TLMS-semi solid matrix

peaks were present, but the intensities became much smaller, indicating that the crystallinity of TLMS reduced in the physical mixture. Crystalline index was calculated to be $33.25 \%$. After preparations of solid dispersion by hot fusion method diffraction peaks of SD4 with reduced intensities were observed. Crystallinity index was calculated to be $24.55 \%$ suggesting the reduction in crystallinity. Reduction in crystallinity of TLMS was found to be due to complex formation between drug and carrier. In contrast, no distinct intensity peaks were observed, confirming the fully amorphous solid dispersions (2).

\section{Scanning Electron Microscopy (SEM)}

Scanning electron micrograph of drug revealed rod shaped crystals. Pluronic-F68 appeared as circular globule like structure without any definite geometry indicating typical amorphous nature of the polymer. SEM micrograph of physical mixture was characterized by rod shaped crystals embedded and deposited on to the surfaces of circular globules of the polymer. However, solid dispersion was marked by the disappearance of the rod shaped crystals (Fig. 3).

\section{Differential scanning calorimetry}

The physical interaction among TLMS and Pluronic-F68 can be better understood by the critical analysis of the thermal behaviour exhibited by pure drug and SD4. The DSC thermogram of pure drug showed a sharp endothermic peak at $272.17{ }^{\circ} \mathrm{C}$ corresponding to its melting point. The sharpness of the thermogram indicated typical crystalline nature of drug particles. Thermogram of Pluronic-F68 showed a single broad peak at $56.46{ }^{\circ} \mathrm{C}$ indicating that the 


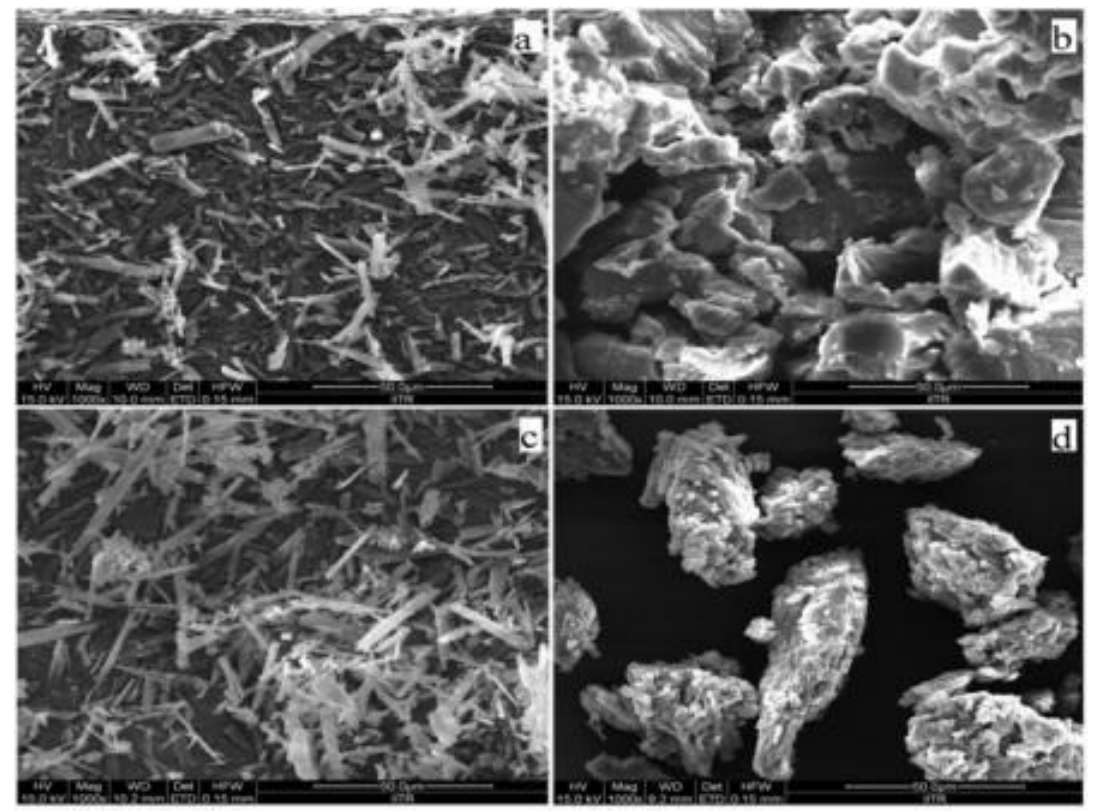

Figure 3 Scanning electron micrograph of a) TLMS, b) Pluronic-F68, c) physical mixture and d) SD4

polymer is amorphous in nature. The solid dispersion showed two peaks, one at $48.23{ }^{\circ} \mathrm{C}$ corresponding to the melting endotherm of Pluronic-F68 and second at $266.47{ }^{\circ} \mathrm{C}$ corresponding to melting endotherm of drug. The endothermic peak of Pluronic-F68 shifted to lower melting point, indicating interaction between the drug and Pluronic-F68. Further, the melting endotherm of drug was characterized by a peak of less ordered state which lacked sharpness (Fig. 2B).

\section{Diffuse reflectance spectroscopy}

DRS spectra of the SD4 (Fig. 4) showed characteristic bands of both drug and polymer without any petite shift in the wave numbers of the peak. However, slight changes in the intensity of peak within permissible limit were documented. This confirms that the changes in the thermogram of pure drug in solid dispersion are due to its interaction with polymer.

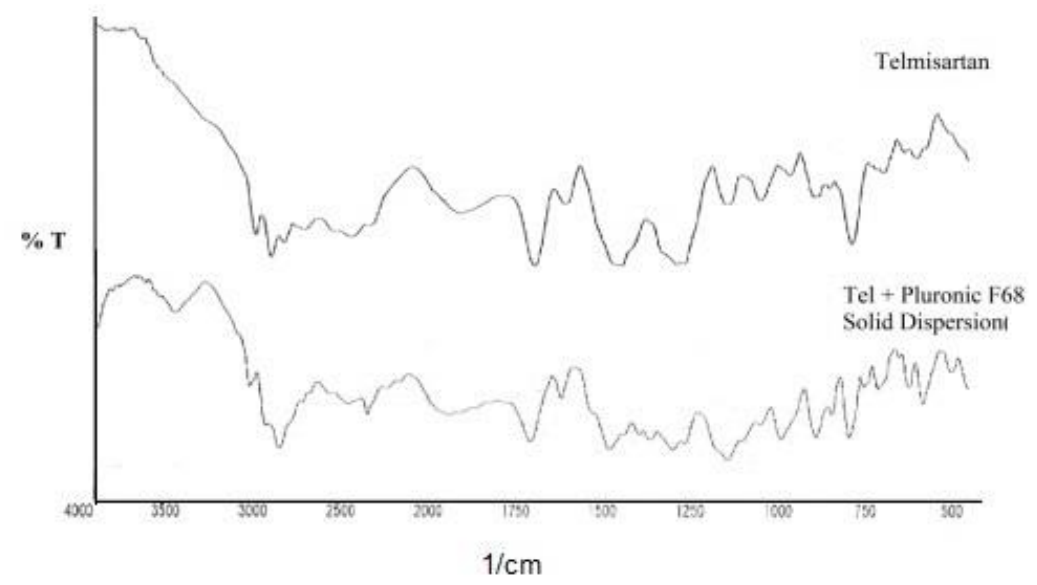

Figure 4 DRS spectra of pure TLMS and SD4 
Table 3 Content determination of centrifuged sample (Dispersal homogeneity test)

\begin{tabular}{lcc}
\hline Batch code & \multicolumn{2}{c}{ Percent drug content } \\
& Samples from top layer & Samples from bottom tube \\
\hline SSC1 & $97.91 \pm 0.62$ & $97.42 \pm 0.48$ \\
SSC2 & $98.42 \pm 0.47$ & $98.13 \pm 0.85$ \\
SSC3 & $96.94 \pm 0.74$ & $96.26 \pm 0.58$ \\
SSC4 & $96.51 \pm 0.86$ & $96.02 \pm 0.74$ \\
SSC5 & $97.04 \pm 0.93$ & $97.73 \pm 0.15$ \\
SSC6 & $98.38 \pm 0.42$ & $98.93 \pm 0.62$ \\
SSC7 & $96.36 \pm 0.74$ & $96.79 \pm 0.14$ \\
\hline
\end{tabular}

Preparation and evaluation of SSCs

Semisolid matrix of optimized solid dispersion formulation (SD4) was formulated and characterized for dispersal homogeneity and in vitro dissolution.

\section{Dispersal homogeneity}

The aqueous dispersion of the semi solid mix when subjected to centrifugation (4000 rpm, $10 \mathrm{~min})$ showed no precipitate/residue at the bottom of the tube or on the surface layer of the test sample. No difference in drug content was evidenced (Table 3 ). Results ensured the uniformity of all the semisolid mix formulations.

\section{In vitro dissolution}

In vitro drug dissolution profiles of SSCs in phosphate buffer, $\mathrm{pH} 7.4$ are shown in Figure 1B. A biphasic dissolution was observed from the profiles: an initial burst phase within 5 min that resulted in \%CDR ranging from $63.92 \%$ to $79.04 \%$. The burst release was followed by a slower dissolution of $80.89 \%$ to $97.49 \%$ up to 20 min. However, a biphasic dissolution of lower magnitude $(10.64 \%$ at $5 \mathrm{~min}$ and 33.95 at $20 \mathrm{~min}$ in phosphate buffer, $\mathrm{pH}$ 7.4) was displayed by SD4 when compared to all SSC formulations. Among the SSCs, maximum drug dissolution $(97.49 \%)$ in phosphate buffer, $\mathrm{pH} 7.4$ up to $20 \mathrm{~min}$ was exhibited by SSC6 comprised of the lowest level of PEG 400 and highest of Gelucire 44/14. However, SSC7 showed the least dissolution of $80.49 \%$ in phosphate buffer, $\mathrm{pH} 7.4$ which consisted of the lowest level of Gelucire 44/14 and the highest amount of PEG 400. Clearly, both Gelucire 44/14 and PEG 400 levels played an important role in enhancing the drug dissolution. The dissolution data were analyzed using the model independent parameters. T80\% and DE $15 \mathrm{~min}$ were utilized to analyze the data, affirming the similar concept of Gelucire 44/14 and PEG 400 level effect on drug dissolution. The time required for $80 \%$ dissolution was found to be least i.e. 5.6 min for SSC6. DE at 15 min was $95.8 \%$ and $78.5 \%$ for SSC6 and SSC7 in phosphate buffer, $\mathrm{pH} 7.4$, respectively. For rest of the formulation intermediate values were obtained that were a reflection of the variation in levels of Gelucire 44/14 and PEG 400. 


\section{Discussion}

Combinational approach based on encapsulation of SD in HPMC capsules have been used in order to enhance the in vitro drug dissolution. SDs prepared were characterized for micromeritic properties. Bulk density (poured and tapped) is defined as an inverse of bulk volume. Thus, lower bulk density support the porous structure and facilitate water ingresment and resulted in rapid disintegration. From the results obtained, it was observed that solid dispersion prepared by various methods exhibited good flow property. Further, findings of $\%$ compressibility index suggested that all the powders possessed good to fair flow properties. Hausner's ratio again proved that all the mixtures acquired good to fair flow properties. The increase in solubility of SD may be the result of reduction of particle size. As the hydrophilic carrier in which the drug is entrapped undergo dissolution, the drug gets molecularly dispersed in the dissolution medium that led to enhanced surface area and hence the dissolution. Further, hydrophilic carrier results in increased wettability of the drug which may also led to increased surface area and reduced agglomeration (24). Reduction in crystalline nature of drug as explained from x-ray diffraction results may also results in increase in solubility and hence the dissolution rate (5). The decrease in solubility with increase in drug: polymer after a certain ratio may be attributed to the self inhibitory effect of Plasdone-S630 above certain concentration. Plasdone-S-630 was known to form a grid like structure around the molecules of drug that restrict the dissolution medium to reach the drug molecules (11). The increase in dissolution of TLMS from SD may be the result of reduction of surface tension that will facilitate the particle partition which may led to the improvement of wetting of hydrophobic TLMS crystals and this provides a favorable environment to enhance the dissolution of drug if release is carrier controlled (26). Further, specificity was measured by analyzing the standard drug solution in the presence of excipients to determine any interference in the percentage recovery. The interference was found to be which is within the acceptable limits. Hence, the excipients do not interfere with the estimates of drug. The sequentially reduced crystallinity has been observed in case of physical mixture and SD4 in XRPD analysis. These transformed results are due to the comparatively less crystalline nature of Pluronic-F68 as compared to pure TLMS. When the drug has been fused in a molten mass of polymer, a smooth coat on the surface of TLMS crystals provides the highest reduction in the intensity of the reflected infra red rays which yields an amorphous solid dispersion. This amorphization positively contributed in the dissolution behavior of the drug as recorded in dissolution studies. SEM results indicated that the drug was uniformly mixed with polymer which resulted into its conversion from crystalline to amorphous state. This might occur due to its physical interaction with PluronicF68 in solid dispersion (SD4). DSC studies suggested that the drug was converted to amorphous state because of the formation of chemical bonds with Pluronic-F68 which may accounts for its enhanced solubility. However, slight changes in the intensity of peak in within permissible limit were documented in DRS spectra. This confirmed the changes in the DSC 
thermogram of pure drug in solid dispersion are due to its interaction with polymer. This may lead to its reduction in crystalline state. Further, semisolid matrix of optimized solid dispersion formulation (SD4) was formulated and characterized for dispersal homogeneity and in vitro dissolution. No difference in drug content was evidenced (Table 3 ). Results ensured the uniformity of all the semisolid mix formulations. From the in vitro dissolution profile, the superiority of the SSCs over SDs was clearly observed which is advantageous for antihypertensive drugs like TLMS, requiring faster onset of action. It may be attributed to the time taken for wetting, dissolution of carrier and development of microenvironment gets reduced in case of SSCs.

Liquid polyethylene glycol is used as water-miscible solvent, but they also possess the property of absorbing moisture from capsule shell (27) that may result in hydration of the filled content and thus reducing the solubility (28). Conversely, Gelucire 44/14 being an ampiphilic excipient possessed surface active properties and is known

\section{References}

1. Goodman, Gilman's., editors. The pharmacological basis of therapeutics: $10^{\text {th }}$ ed. Mc Graw-Hill, Medical Publishing Divison; 2001.

2. Patel B, Parikh RH, Swarnkar D. Enhancement of dissolution of telmisartan through use of solid dispersion techniquesurface solid dispersion. J Pharm Bioallied Sci 2012; 4: S64-8.

3. Kaur M, Bhatia RK, Pissurlenkar RR, Coutinho EC, Jain UK, Katare OP, et al. Telmisartan complex augments solubility, dissolution and drug delivery in prostate cancer cells. Carbohydrate Polymers 2014; 101: 614-22.

4. Zhang Y, Zhuangzhi Z, Jiang T, Zhang J, Wang Z, Wang S. Spherical mesoporous silica nanoparticles for loading and release of the poorly water-soluble drug to enhance the drug solubility either by micellar solubilization of drug or improving the wetting characteristic or by both process (29). Thus, as a result of increased hydrophilicity, the faster drug dissolution was obtained. Thus, SSC6 comprising lowest level of PEG 400 and highest of Gelucire 44/14 shows the highest dissolution.

\section{Conclusion}

The present study revealed that solubility of the antihypertensive telmisartan was successfully enhanced by solid dispersion. The conversion of solid dispersion into semi solid matrix filled in HPMC capsule further results in increase solubility and hence dissolution rate with approximately zero lag time. Thus, SSC present an excellent approach for enhancing the solubility and hence the bioavailability of the poorly water soluble drug telmisartan.

\section{Conflict of Interest}

The authors declared no conflict of interest.

telmisartan. J Controlled Rel 2010; 145: 257-63.

5. Bhise S, Mathure D, Patil MVK, Patankar RD. Solubility enhancement of antihypertensive agent by solid dispersion technique. Int J Pharm Life Sci 2011; 2: 791-6.

6. Bajaj A, Rao MRP, Pardeshi A, Sali D. Nanocrystallization by evaporative antisolvent technique for solubility and bioavailability enhancement of telmisartan. AAPS PharmSciTech 2012; 13: 1331-40.

7. Nikghalb LA, Singh G, Singh G, Kahkeshan KM. Solid Dispersion: Methods and Polymers to increase the solubility of poorly soluble drugs. J Appl Pharm Sci 2012; 2: 170-5.

8. Walker SE, Ganley JA, Bedford K, Eaves $\mathrm{T}$. The filling of molten and thixotropic formulations into hard gelatin capsules. $\mathrm{J}$ Pharm Pharmacol 1980; 32: 389-93 
9. Kumar M, Sistla R, Shastri NR. Modulating drug release profiles by lipid semisolid matrix formulations for BCS class II drugan in vitro and in vivo study. Drug Deliv 2015; 22: 418-26.

10. Alladi S, Shastri NR. Semi solid matrix formulations of meloxicam and tenoxicam: an in vitro and in vivo evaluation. Arch Pharm Res 2015; 38: 801-12.

11. Sun Y, Rui Y, Wenliang Z, Tang X. Nimodipine semi-solid capsules containing solid dispersion for improving dissolution. Int J Pharm 2008; 359: 144-9.

12. Tyagi VK, Singh D, Pathak K. Semisolid matrix-filled hard gelatin capsules for rapid dissolution of amlodipine besilate: Development and assessment. J Adv Pharm Tech Res 2013; 4: 42-9.

13. Ghosh A. Veg or non-veg. The Ind Exp 2015; 5: 1-12.

14. Al-Tabakha MM. HPMC capsules: current status and future prospects. J Pharm Pharmaceut Sci 2010; 13: 428-42.

15. Ku MS, Lu Q, Li W, Chen Y. Performance qualification of a new hypermellose capsule. Int J Pharm 2010; 386: 30-41.

16. Amanda A. Properties of capsule shells made from hydroxypropyl methylcellulose (Hypromellose). 2010. pp. 1-2. Thesis (Doctoral). http://sure.sunderland.ac.uk. id/eprint/3779. Accessed on 1/11/2016.

17. Srividya B, Chaudhary C, Reddy CSP. Capsules and its technology: an overview. Int J Pharm Drug Anal 2014; 2: 727-33.

18. Aulton ME. The Design and Manufacturing of Medicines, 3rd ed.; Harcourt Publishers Limited: Philadelphia, PA, USA, 2007; pp. 16-31, 355-7.

19. Sinko PJ. Martin's Physical Pharmacy and Pharmaceutical Sciences, 5th ed.; Wolters Kluwer (India) Pvt. Ltd.: New Delhi, India, 2008; pp. 533-59.

20. Shah M, Pathak K. Development and statistical optimization of sold lipid nanoparticles of simvastatin by using $2^{3}$ full-factorial design. AAPS PharmSciTech 2010; 11: 489-96.
21. Singh G, Chhabra G, Pathak K. Dissolution behaviour and thermodynamic stability of fused-sugar dispersions of a poorly water soluble drug. Disso Tech 2010; 1: 62-70.

22. Khan MA., Akhtar N, Sharma V, Pathak K. Product development studies on sonocrystallized curcumin for the treatment of gastric cancer. Pharmaceutics 2015; 7 : 43-63.

23. Dahan A, Miller JM, Amidon GL. Prediction of solubility and permeability class membership: Provisional BCS classification of the world's top oral drugs. AAPS J 2009; 11: 740-6.

24. Sharma A, Jain CP. Solid dispersion: A promising technique to enhance solubility of poorly water soluble drug. Int J Drug Deliv 2011; 3: 149-70.

25. El- Badryl M, Fetih G, Fathy M. Improvement of solubility and dissolution rate of indomethacin by solid dispersions in Gelucire 50/13 and PEG4000. Saudi Pharm J 2009; 17: 217-25

26. Dora CP, Singh SK, Kumar S, Datusalia AK, Deep A. Development and characterization of nanoparticles of glibenclamide by solvent displacement method. Acta Pol Pharm 2010; 67: 283-90.

27. Rowe RC, Sheskey PJ, Own SC. Handbook of Pharmaceutical Excipients. $5^{\text {th }}$ ed. Published by Pharmaceutical Press \& American Pharmaceutical Associations, USA, 2006.

28. Serajuddin AT, Sheen PC, Augustine MA. Water migration from soft gelatin capsule shell to fill material and its effect on drug solubility. J Pharm Sci 1986; 75: 62-4.

29. Karatas A, Yuksel N, Baykara T. Improved solubility and dissolution rate of piroxicam using Gelucire 44/14 and labrasol. Farmaco 2005; 60: 777-82. 